\title{
Eventos - 2008
}

European League Against Rheumatism (Eular) Annual 2008 Congress

Data: 11 a 14 de junho de 2008

Local: Paris, França

XXVII Congresso Uruguaio de Reumatologia

Data: 25 a 28 de junho

Local: Montevidéu, Uruguai

XV Congresso de la Liga Panamericana de Asociaciones de Reumatologia - PANLAR

Data: 13 a 16 de agosto

Local: Guatemala

Contato: www.panlar2008.agreuma.org

\section{$12^{\text {th }}$ World Congress on Pain}

Data: 17 a 22 de agosto

Local: Glasgow, Escócia

VI Congresso de Auto-Imunidade

Data: 3 a 7 de setembro

Local: Porto, Portugal

Contato: www.kenes.com/autoimmunity

XXX Congresso da Sociedade Americana de Metabolismo Ósseo - ASBMR

Data: 12 a 16 de setembro

Local: Montreal, Canadá

Contato: www.asbmr.org

$15^{\text {th }}$ PReS Congress - European Paediatric

Rheumatology Society

Data: 14 a 17 de setembro

Local: Londres, Inglaterra
Curso de Revisão de Reumatologia para Clínicos

Data: 17 a 18 de setembro

Local: São Paulo, SP

Contato: www.reumatologiasp.com.br

XXVII Congresso Brasileiro de Reumatologia

Data: 17 a 20 de setembro

Local: Maceió, AL

Contato: www.reumatologia.com.br/eventos

72 Congresso do Colégio Americano de

Reumatologia (ACR)

Data: 24 a 29 de outubro

Local: São Francisco, EUA

Contato: www.rheumatology.org

Congresso Chileno de Reumatologia 2008

Data: 12 a 15 de novembro

Local: Puerto Varas, Chile

41 ongresso Argentino de Reumatologia

Data: 19 a 22 de novembro

Local: Mar del Plata, Argentina

\section{Congresso Mundial de Osteoporose}

Data: 3 a 7 de dezembro

Local: Bangkok, Tailândia

Contato: www.iofbonehealth.org

\section{ERRATA}

ReVista Brasileira de Reumatologia

JAN/FEV $2008 \bullet$ VOLUME $48 \bullet$ № 1

Pg. 31

Onde se lê:

1. Conselheiro do Conselho Regional de Medicina do Estado de São Paulo (Cremesp) e membro da Comissão de Ética e Defesa Profissional da Sociedade Brasileira de Reumatologia (SBR) e Sociedade Portuguesa de Reumatologia (SPR).

Leia-se:

1. Conselheiro do Conselho Regional de Medicina do Estado de São Paulo (Cremesp) e membro da Comissão de Ética e Defesa Profissional da Sociedade Brasileira de Reumatologia (SBR) e Sociedade Paulista de Reumatologia (SPR). 